\title{
A Fuzzy Index Tracking Portfolio Selection Model*
}

\author{
Yong Fang and Shou-Yang Wang ${ }^{\star \star}$ \\ Institute of Systems Science, \\ Academy of Mathematics and Systems Science, \\ Chinese Academy of Sciences, Beijing 100080, China \\ yfang@amss.ac.cn \\ swang@iss.ac.cn
}

\begin{abstract}
The investment strategies can be divided into two classes: passive investment strategies and active investment strategies. An index tracking investment strategy belongs to the class of passive investment strategies. The index tracking error and the excess return are considered as two objective functions, a bi-objective programming model is proposed for the index tracking portfolio selection problem. Furthermore, based on fuzzy decision theory, a fuzzy index tracking portfolio selection model is also proposed. A numerical example is given to illustrate the behavior of the proposed fuzzy index tracking portfolio selection model.
\end{abstract}

\section{Introduction}

In financial markets, the investment strategies can be divided into two classes: passive investment strategies and active investment strategies. Investors who are adopting active investment strategies carry out securities exchange actively so that they can find profit opportunity constantly. Active investors take it for granted that they can beat markets continuously. Investors who are adopting passive investment strategies consider that the securities market is efficient. Therefore they cannot go beyond the average level of market continuously. Index tracking investment is a kind of passive investment strategy, i.e., investors purchase all or some securities which are contained in a securities market index and construct an index tracking portfolio. The securities market index is considered as a benchmark. The investors want to obtain a similar return as that of the benchmark through the index tracking investment.

In 1952, Markowitz [6, 7] proposed the mean variance methodology for portfolio selection. It has served as a basis for the development of modern financial theory over the past five decades. Konno and Yamazaki [5] used the absolute deviation risk function to replace the risk function in Markowitz's model to formulate a mean absolute deviation portfolio optimization model. Roll 8 used

\footnotetext{
* Supported by the National Natural Science Foundation of China under Grant No. 70221001 .

** Corresponding author.
} 
the sum of the squared deviations of returns on a replicating portfolio from benchmark as the tracking error and proposed a mean variance index tracking portfolio selection model. Clarke, Krase and Statman [2] defined a linear tracking error which is the absolute deviation between the managed portfolio return and the benchmark portfolio return. Based on the linear objective function in which absolute deviations between portfolio and benchmark returns are used, Rudolf, Wolter and Zimmermann [9] proposed four alternative definitions of a tracking error. Furthermore, they gave four linear optimization models for index tracking portfolio selection problem. Consiglio and Zenios [3] and Worzel, Vassiadou-Zeniou and Zenios [11] studied the tracking indexes of fixed-income securities problem. In this paper, we will use the excess return and the linear tracking error as objective functions and propose a bi-objective programming model for the index tracking portfolio selection problem. Furthermore, we use fuzzy numbers to describe investors' vague aspiration levels for the excess return and the tracking error and propose a fuzzy index tracking portfolio selection model.

The paper is organized as follows. In Section 2, we present a bi-objective programming model for the index tracking portfolio selection problem. In Section 3, regarding investors' vague aspiration levels for the excess return and linear tracking error as fuzzy numbers, we propose a fuzzy index tracking portfolio selection model. In Section 4, a numerical example is given to illustrate the behavior of the proposed fuzzy index tracking portfolio selection model. Some concluding remarks are given in Section 5 .

\section{Bi-objective Programming Model for Index Tracking Portfolio Selection}

In this paper, we assume that an investor wants to construct a portfolio which is required to track a securities market index. The investor allocates his/her wealth among $n$ risky securities which are component stocks contained in the securities market index. We introduce some notations as follows.

$r_{i t}$ : the observed return of security $i(i=1,2, \cdots, n)$ at time $t(t=1,2, \cdots, T)$;

$x_{i}$ : the proportion of the total amount of money devoted to security $i(i=$ $1,2, \cdots, n)$

$I_{t}$ : the observed securities market index return at time $t(t=1,2, \cdots, T)$.

Let $x=\left(x_{1}, x_{2}, \cdots, x_{n}\right)$. Then the return of portfolio $x$ at time $t(t=$ $1,2, \cdots, T)$ is given by

$$
R_{t}(x)=\sum_{i=1}^{n} r_{i t} x_{i} .
$$

An excess return is the return of index tracking portfolio $x$ above the return on the index. The excess return of portfolio $x$ at time $t(t=1,2, \cdots, T)$ is given by

$$
E_{t}(x)=R_{t}(x)-I_{t}
$$


The expected excess return of index tracking portfolio $x$ is given by

$$
E(x)=\sum_{t=1}^{T} \frac{1}{T}\left(R_{t}(x)-I_{t}\right) .
$$

Roll [8] used the sum of squared deviations between the portfolio and benchmark returns to measure the tracking error of index tracking problem. Rudolf, Wolter and Zimmermann [9] used linear deviations instead of squared deviations to give four definitions of the linear tracking errors. We adopt the tracking error based on the mean absolute downside deviations to formulate the index tracking portfolio selection model in this paper. The tracking error based on the mean absolute downside deviations can be expressed as

$$
T_{D M A D}(x)=\sum_{t=1}^{T} \frac{1}{T}\left|\min \left\{0, R_{t}(x)-I_{t}\right\}\right| .
$$

Generally, in the index tracking portfolio selection problem, the track error and the excess return are two important factors which are considered by investors. An investor tries to maximize the expected excess return. At the same time, the investor hopes that the return of portfolio equals the return of the index approximatively to some extent in the investment horizon. Hence, the expected excess return and the tracking error can be considered as two objective functions of the index tracking portfolio selection problem.

In many financial markets, the securities are no short selling. So we add the following constraints:

$$
x_{1}, x_{2}, \cdots, x_{n} \geq 0, i=1,2, \cdots, n .
$$

We assume that the investor pursues to maximize the excess return of portfolio and to minimize the tracking error under the no short selling constraint. The index tracking portfolio selection problem can be formally stated as the following bi-objective programming problem:

$$
\begin{aligned}
\text { (BP) } \max & E(x) \\
\min & T_{D M A D}(x) \\
\text { s.t. } & \sum_{i=1}^{n} x_{i}=1, \\
& x_{1}, x_{2}, \cdots, x_{n} \geq 0, i=1,2, \cdots, n .
\end{aligned}
$$

The problem (BP) can be reformulated as a bi-objective linear programming problem by using the following technique. Note that

$$
|\min \{0, a\}|=\frac{1}{2}|a|-\frac{1}{2} a
$$

for any real number $a$. Thus, by introducing auxiliary variables $b_{t}^{+}, b_{t}^{-}, t=$ $1,2, \cdots, T$ such that

$$
b_{t}^{+}+b_{t}^{-}=\frac{\left|R_{t}(x)-I_{t}\right|}{2}
$$




$$
\begin{gathered}
b_{t}^{+}-b_{t}^{-}=\frac{R_{t}(x)-I_{t}}{2}, \\
b_{t}^{+} \geq 0, \quad b_{t}^{-} \geq 0, t=1,2, \cdots, T,
\end{gathered}
$$

we may write

$$
T_{D M A D}(x)=\sum_{t=1}^{T} \frac{2 b_{t}^{-}}{T} .
$$

Hence, we may rewrite problem (BP) as the following bi-objective linear programming problem:

$$
\begin{aligned}
& \text { (BLP) } \max E(x) \\
& \min \sum_{t=1}^{T} \frac{2 b_{t}^{-}}{T} \\
& \text { s.t. (1), (2) and all constraints of (BP). }
\end{aligned}
$$

Thus the investor may get the index tracking investment strategies by computing efficient solutions of (BLP). One can use one of the existing algorithms of multiple objective linear programming to solve it efficiently.

\section{$3 \quad$ Fuzzy Index Tracking Portfolio Selection Model}

In an investment, the knowledge and experience of experts are very important in an investor's decision-making. Based on experts' knowledge, the investor may decide his/her levels of aspiration for the expected excess return and the tracking error of index tracking portfolio. In [10, Watada employed a non-linear $S$ shape membership function, to express aspiration levels of expected return and of risk which the investor would expect and proposed a fuzzy active portfolio selection model. The $S$ shape membership function is given by:

$$
f(x)=\frac{1}{1+\exp (-\alpha x)} .
$$

In the bi-objective programming model of index tracking portfolio selection proposed in Section 2, the two objectives, the expected excess return and the tracking error, are considered. Since the expected excess return and the tracking error are vague and uncertain, we use the non-linear $S$ shape membership functions proposed by Watada to express the aspiration levels of the expected excess return and the tracking error.

The membership function of the expected excess return is given by

$$
\mu_{E}(x)=\frac{1}{1+\exp \left(-\alpha_{E}\left(E(x)-E_{M}\right)\right)},
$$

where $E_{M}$ is the mid-point where the membership function value is 0.5 and $\alpha_{E}$ can be given by the investor based on his/her own degree of satisfaction for the 


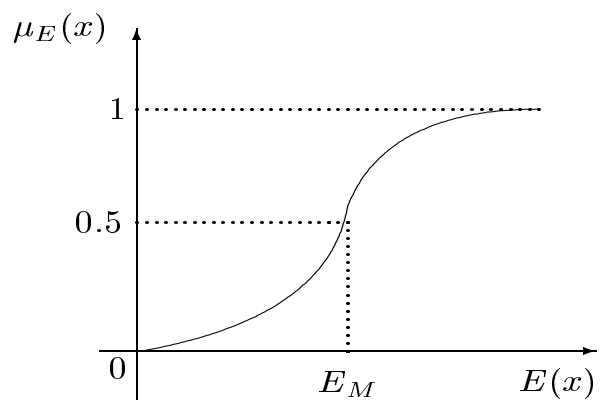

Fig. 1. Membership function of the goal for expected excess return

expected excess return. Figure 1 shows the membership function of the goal for the expected excess return.

The membership function of the tracking error is given by

$$
\mu_{T}(x)=\frac{1}{1+\exp \left(\alpha_{T}\left(T_{D M A D}(x)-T_{M}\right)\right)},
$$

where $T_{M}$ is the mid-point where the membership function value is 0.5 and $\alpha_{T}$ can be given by the investor based on his/her own degree of satisfaction regarding the level of tracking error. Figure 2 shows the membership function of the goal for the tracing error.

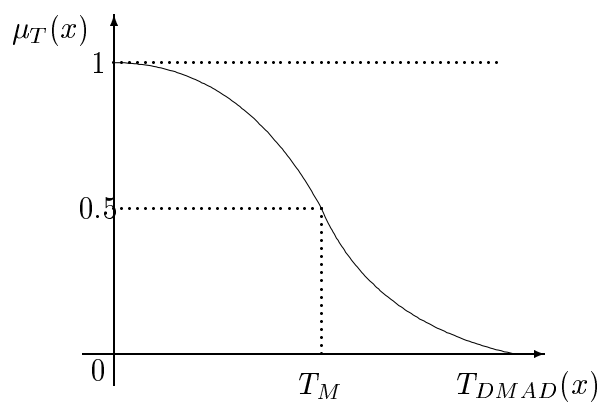

Fig. 2. Membership function of the goal for tracking error

Remark1: $\alpha_{E}$ and $\alpha_{T}$ determine the shapes of membership functions $\mu_{E}(x)$ and $\mu_{T}(x)$ respectively, where $\alpha_{E}>0$ and $\alpha_{T}>0$. The larger parameters $\alpha_{E}$ and $\alpha_{T}$ get, the less their vagueness becomes.

According to Bellman and Zadeh's maximization principle [1, we can define

$$
\lambda=\min \left\{\mu_{E}(x), \mu_{T}(x)\right\} .
$$

The fuzzy index tracking portfolio selection problem can be formulated as follows: 


$$
\begin{aligned}
\text { (FP) } \max & \lambda \\
\text { s.t. } & \mu_{E}(x) \geq \lambda \\
& \mu_{T}(x) \geq \lambda, \\
& \text { and all constraints of (BLP). }
\end{aligned}
$$

Let $\eta=\log \frac{1}{1-\lambda}$, then $\lambda=\frac{1}{1+\exp (-\eta)}$. The logistic function is monotonously increasing, so maximizing $\lambda$ makes $\eta$ maximize. Therefore, the above problem can be transformed to an equivalent problem as follows:

$$
\begin{aligned}
\text { (FLP) } \max & \eta \\
\text { s.t. } & \alpha_{E}\left(E(x)-E_{M}\right)-\eta \geq 0, \\
& \alpha_{T}\left(T_{D M A D}(x)-T_{M}\right)+\eta \leq 0, \\
& \text { and all constraints of }(\mathrm{BLP}),
\end{aligned}
$$

where $\alpha_{E}$ and $\alpha_{T}$ are parameters which can be given by the investor based on his/her own degree of satisfaction regarding the expected excess return and the tracking error.

(FLP) is a standard linear programming problem. One can use one of several algorithms of linear programming to solve it efficiently, for example, the simplex method.

Remark2: The non-linear $S$ shape membership functions of the two factors may change their shape according to the parameters $\alpha_{E}$ and $\alpha_{T}$. Through selecting the values of these parameters, the aspiration levels of the two factors may be described accurately. On the other hand, different parameter values may reflect different investors' aspiration levels. Therefore, it is convenient for different investors to formulate investment strategies by using the proposed fuzzy index tracking portfolio selection model.

\section{$4 \quad$ Numerical Example}

In this section, we will give a numerical example to illustrate the proposed fuzzy index tracking portfolio selection model. We suppose that the investor considers Shanghai 180 index as the tracking goal. We choose thirty component stocks form Shanghai 180 index as the risky securities. We collect historical data of the thirty stocks and Shanghai 180 index from January, 1999 to December, 2002. The data are downloaded from the web-site www.stockstar.com. We use one month as a period to get the historical rates of returns of forty eight periods.

The values of the parameters $\alpha_{E}, \alpha_{T}, E_{M}$ and $T_{M}$ can be given by the investor according his/her aspiration levels for the expected excess return and the tracking error. In the example, we assume that $\alpha_{E}=500, \alpha_{T}=1000$, $E_{M}=0.010$ and $T_{M}=0.009$. Using the historical data, we get an index tracking portfolio selection strategy by solving (FLP). All computations were carried out on a WINDOWS PC using the LINDO solver. Table 1 shows the obtained expected excess return and tracking error of portfolio by solving (FLP). Table 2 shows the investment ratio of the obtained fuzzy index tracking portfolio. 
Table 1. Membership grade $\lambda$, obtained expected excess return and obtained tracking error

\begin{tabular}{cccc}
\hline$\lambda$ & $\eta$ & excess return & tracking error \\
\hline 0.9431 & 2.8095 & 0.0152 & 0.0062 \\
\hline
\end{tabular}

Table 2. Investment ratio of the obtained fuzzy index tracking portfolio

\begin{tabular}{lcccccccccc}
\hline Stock & 1 & 2 & 3 & 4 & 5 & 6 & 7 & 8 & 9 & 10 \\
\hline Ratio & 0.0000 & 0.0000 & 0.0620 & 0.0254 & 0.0000 & 0.0408 & 0.0180 & 0.1389 & 0.0324 & 0.0082 \\
\hline Stock & 11 & 12 & 13 & 14 & 15 & 16 & 17 & 18 & 19 & 20 \\
\hline Ratio & 0.1440 & 0.1488 & 0.0130 & 0.0000 & 0.0000 & 0.0000 & 0.1889 & 0.0000 & 0.0000 & 0.0000 \\
\hline Stock & 21 & 22 & 23 & 24 & 25 & 26 & 27 & 28 & 29 & 30 \\
\hline Ratio & 0.0276 & 0.0000 & 0.0000 & 0.0124 & 0.1001 & 0.0000 & 0.0395 & 0.0000 & 0.0000 & 0.0000 \\
\hline
\end{tabular}

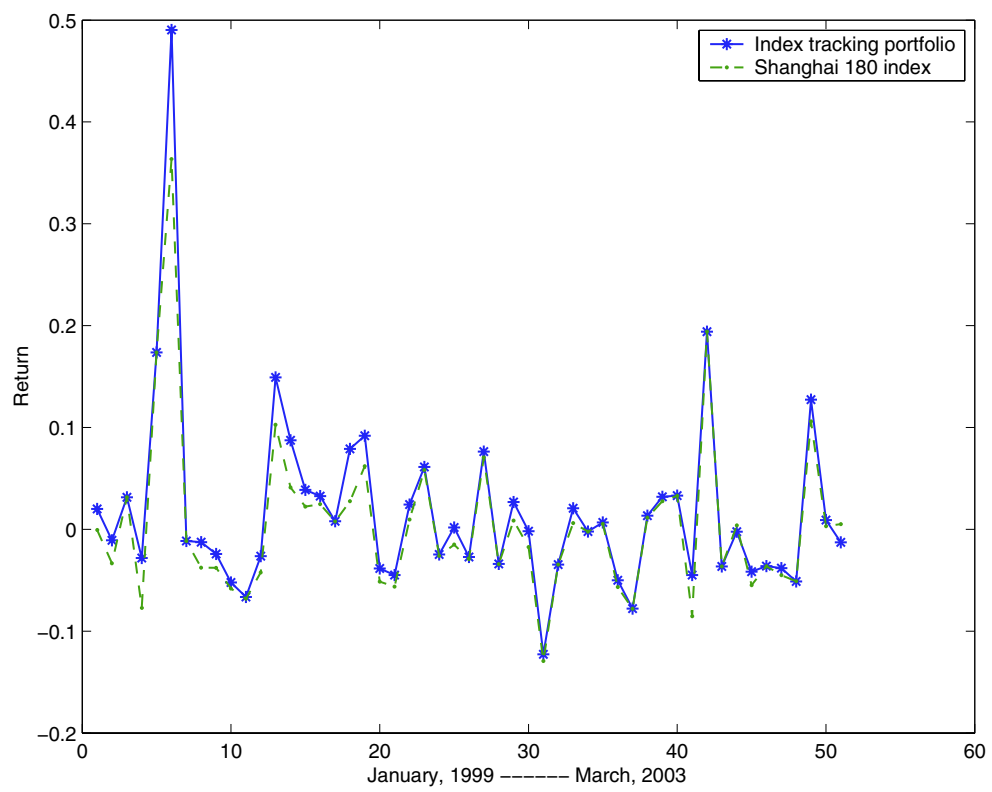

Fig. 3. The deviations between the returns of the obtained index tracking portfolio and the returns on the benchmark Shanghai 180 index

Figure 3 shows the deviations between the returns of the obtained index tracking portfolio and the returns on the benchmark Shanghai 180 index for each month from January, 1999 to March, 2003. From Figure 3, we can find that the obtained fuzzy index portfolio by solving (FLP) tracks Shanghai 180 index efficiently. 


\section{Conclusion}

Regarding the expected excess return and the tracking error as two objective functions, we have proposed a bi-objective programming model for the index tracking portfolio selection problem. Furthermore, investors' vague aspiration levels for the excess return and the tracking error are considered as fuzzy numbers. Based on fuzzy decision theory, we have proposed a fuzzy index tracking portfolio selection model. An example is given to illustrate that the proposed fuzzy index tracking portfolio selection model. The computation results show that the proposed model can generate a favorite index tracking portfolio strategy according to the investor's satisfactory degree.

\section{References}

1. Bellman, R., Zadeh, L.A.: Decision Making in a Fuzzy Environment. Management Science 17 (1970) 141-164.

2. Clarke, R.G., Krase, S., Statman, M.: Tracking Errors, Regret, and Tactical Asset Allocation. Journal of Portfolio Management 20 (1994) 16-24.

3. Consiglio, A., Zenios, S.A.: Integrated Simulation and Optimization Models for Tracking International Fixed Income Indices. Mathematical Programming 89 (2001) 311-339.

4. Fang, Y., Wang, S.Y.: Fuzzy Portfolio Optimization: Theory and Methods. Tsinghua University Press, Beijing, 2005.

5. Konno, H., Yamazaki, H.: Mean Absolute Portfolio Optimization Model and Its Application to Tokyo Stock Market. Management Science 37(5) (1991) 519-531.

6. Markowitz, H.M.: Portfolio Selection. Journal of Finance 7 (1952) 77-91.

7. Markowitz, H.M.: Portfolio Selection: Efficient Diversification of Investment. John Wiley \& Sons, New York, 1959.

8. Roll, R.: A Mean Variance Analysis of Tracking Error - Minimizing the volatility of Tracking Error will not Produce a More Efficient Managed Portfolio. Journal of Portfolio Management 18 (1992) 13-22.

9. Rudolf, M., Wolter, H.J., Zimmermann, H.: A Linear Model for Tracking Error Minimization. Journal of Banking and Finance 23 (1999) 85-103.

10. Watada, J.: Fuzzy Portfolio Model for Decision Making in Investment. In: Yoshida, Y. (eds.): Dynamical Asspects in Fuzzy Decision Making. Physica-Verlag, Heidelberg (2001) 141-162.

11. Worzel, K.J., Vassiadou-Zeniou, C., Zenios, S.A.: Integrated Simulation and Optimization Models for Tracking Indices of Fixed-income Securities. Opreations Research 42 (1994) 223-233. 